\title{
FGF-2 controls the differentiation of resident cardiac precursors into functional cardiomyocytes
}

\author{
Nathalie Rosenblatt-Velin, ${ }^{1}$ Mario G. Lepore, ${ }^{1}$ Cristina Cartoni, ${ }^{1}$ Friedrich Beermann, ${ }^{2}$ \\ and Thierry Pedrazzini ${ }^{1}$
}

1Department of Medicine, University of Lausanne Medical School, Lausanne, Switzerland.

${ }^{2}$ Swiss Institute for Experimental Cancer Research, Epalinges, Switzerland.

\begin{abstract}
Recent evidence suggests that the heart possesses a greater regeneration capacity than previously thought. In the present study, we isolated undifferentiated precursors from the cardiac nonmyocyte cell population of neonatal hearts, expanded them in culture, and induced them to differentiate into functional cardiomyocytes. These cardiac precursors appear to express stem cell antigen-1 and demonstrate characteristics of multipotent precursors of mesodermal origin. Following infusion into normal recipients, these cells home to the heart and participate in physiological and pathophysiological cardiac remodeling. Cardiogenic differentiation in vitro and in vivo depends on FGF-2. Interestingly, this factor does not control the number of precursors but regulates the differentiation process. These findings suggest that, besides its angiogenic actions, FGF- 2 could be used in vivo to facilitate the mobilization and differentiation of resident cardiac precursors in the treatment of cardiac diseases.
\end{abstract}

\section{Introduction}

Cardiac diseases, in particular heart failure, are major causes of mortality and morbidity $(1,2)$. Current therapies do not address adequately the loss of contractile tissues in the diseased heart. This damage accounts for a large part of the deterioration of cardiac functions. On the other hand, recent lines of evidence suggest that the heart demonstrates a greater degree of regeneration than previously thought. This is supported by observations indicating that cardiomyocytes could be replaced continually in the heart through a process involving cellular aging, senescence, and death as well as myocardial cell replication (3). Although this alternative view challenges the strong belief that cardiomyocytes are terminally differentiated cells that do not divide, it has been fueled by the discovery, in many adult organs, of pluripotent progenitors with a capacity to differentiate into a variety of cell types $(4,5)$.

Indeed, cardiomyocytes can be produced in vitro and in vivo from adult pluripotent cells. For example, HSCs or endothelial progenitor cells demonstrate an ability to differentiate into cardiomyocytes (6-9). However, whether or not stem cells from peripheral origin can represent a valuable source of progenitors for replacement therapy in cardiac diseases remains to be established. In reality, the rate of commitment to the cardiogenic lineage after adoptive transfer in vivo is low, and the exact contribution of cell fusion in the engraftment process needs to be clarified (10-14). Therefore, an alternative approach to inducing cardiac healing would consist in the mobilization and differentiation of resident stem cells in the heart. However, to insure a tight control of these signaling events, we need to carefully identify the humoral factors

Nonstandard abbreviations used: BMP, bone morphogenetic protein; EMT, epithelial-to-mesenchymal transformation; MLC, myosin light chain; NMC, nonmyocyte cell; Sca-1, stem cell antigen-1.

Conflict of interest: The authors have declared that no conflict of interest exists.

Citation for this article: J. Clin. Invest. 115:1724-1733 (2005).

doi:10.1172/JCI23418. that are released from the cardiac microenvironment, and which might regulate the fate and cardiogenic conversion of undifferentiated progenitors (15).

Among others, FGF-2 has been implicated in cell proliferation, survival, and differentiation $(16,17)$. It is a member of the family of heparin-binding growth factors that bind tyrosine kinase receptors. In the heart, FGF-2 expression was shown to be upregulated after cardiac injury, such as ischemia/reperfusion, or in the process of cardiac remodeling (18). Along these lines, this factor has been reported to confer cardioprotection in a variety of animal models (19-21) and has been shown to be beneficial for treating ischemic conditions in several clinical trials (22-25). It is also important to note that FGF-2 plays a role in driving mesodermal cells to the cardiogenic lineage during embryogenesis (26-28). Finally, FGF-2 was shown to be critical for the proliferation of the hemangioblast, the common progenitor of hematopoietic and endothelial cells (29-31). Therefore, in an attempt to assess the capacity of the heart to produce newly formed myocytes, which could be activated and recruited for cardiac repair, we initiated a series of experiments aimed at isolating undifferentiated cardiac precursors from the neonatal and the adult heart. Second, we established the conditions for the expansion and differentiation of these cells in vitro and in vivo. And finally, we focused our attention on signals provided by FGF-2.

\section{Results}

Identification, isolation, and differentiation of cardiac precursors. In many organ systems, precursor cells have been identified based on the expression of stem cell antigen-1 (Sca-1). In the neonatal heart of WT mice, Sca- $1^{+}$cells were readily detected by immunostaining (Figure 1A). In the adult, Sca-1 expression was also observed, and associated with a nonmyocyte population (Figure 1, A and B). Moreover, in the adult, Sca-1 was overexpressed during compensatory cardiac hypertrophy that developed secondary to renovascular hypertension (2-kidney 1-clip model; ref. 32). 
A

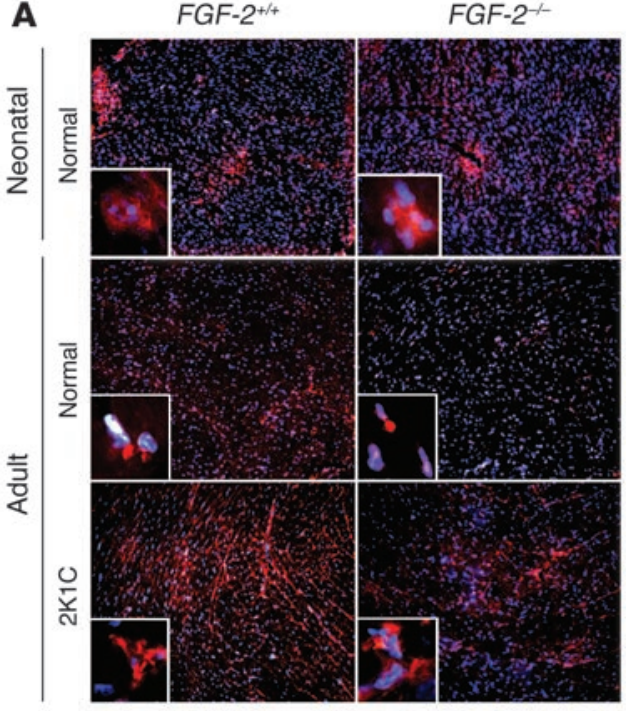

C

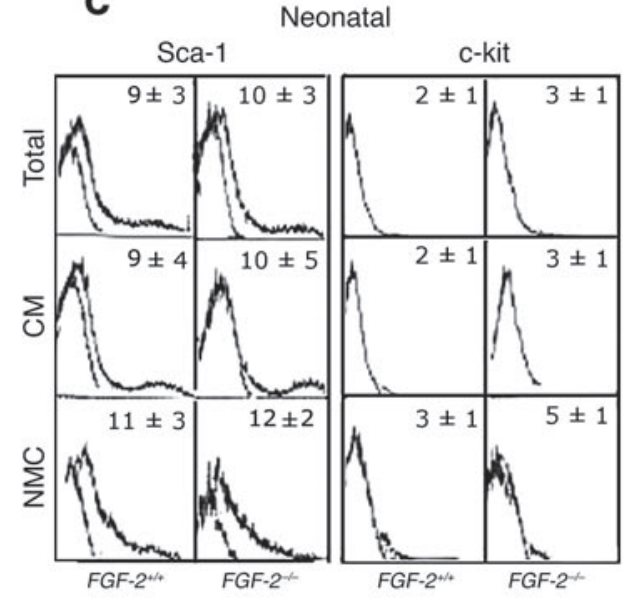

B

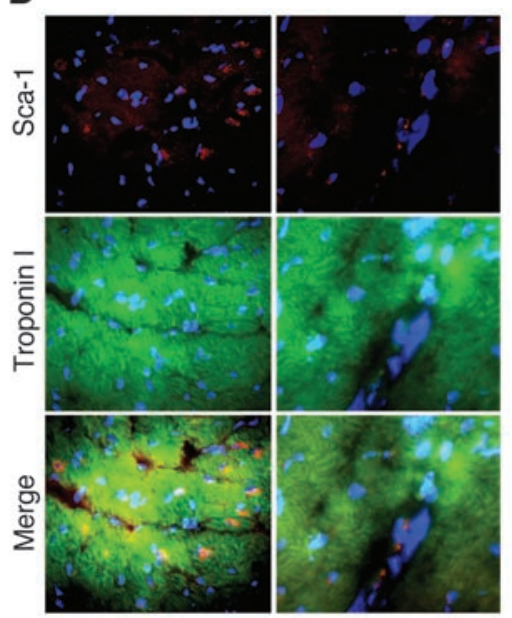

D

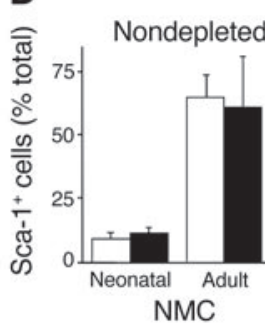

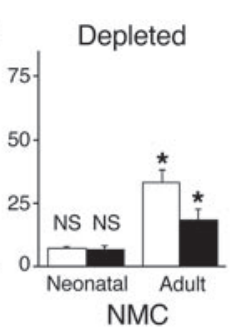

\section{Figure 1}

Sca-1 expression in cardiac tissues from WT $\left(F G F-2^{+/+}\right)$or FGF-2-deficient $\left(F G F-2^{-/-}\right)$ mice. (A) Sca-1 immunostaining in heart sections from $\mathrm{FGF}-2^{+/+}$and $\mathrm{FGF}-2^{-/-}$neonatal and adult mice with or without renovascular hypertension and cardiac hypertrophy (2-kidney 1-clip model [2K1C]). Original magnification, $\times 20$ and $\times 63$ (insets). (B) Immunodetection of Sca-1 and troponin I in the hearts of adult $\mathrm{FGF}-2^{+/+}$mice. Original magnification, $\times 40$ (left panels), $\times 63$ (right panels). (C) Percentage of Sca- $1^{+}$and c-kit ${ }^{+}$cells as determined by FACS analysis in total heart cells (Total) and in the cardiomyocyte $(\mathrm{CM})$ and nonmyocyte cell (NMC) fractions from $\mathrm{FGF}-2^{+/+}$and $\mathrm{FGF}-2^{-/-}$ mice $(n=5)$. (D) Percentage of Sca-1+ cells as determined by FACS analysis in neonatal or adult NMCs isolated from $F G F-2^{+/+}$(white bars) and $F G F-2^{-/-}$mice (black bars). NMCs were either depleted or not depleted of cells expressing CD45, CD31, CD34, Flk-1, CD3, CD4, CD8, B220, TER119, Mac-1, and Gr-1. ${ }^{\star} P<0.05$; NS, not significant as compared with the corresponding nondepleted group; $n=3-6$.
Neonatal hearts were then isolated, and a cardiac single-cell suspension was obtained using enzymatic digestion. Approximately $10 \%$ of the whole cardiac population expressed Sca-1 (Figure 1C, Total). These cells were further separated into cardiomyocyte and nonmyocyte cell (NMC) fractions. The percentage of cells expressing the stem cell marker in these different fractions was equivalent to that observed in the starting population (Figure 1C). Furthermore, about $2-3 \%$ of the cells were positive for c-kit expression. Double-positive cells represented only $0.5 \%$ of NMCs (data not shown). As compared with the neonatal heart, a greater number of adult cardiac NMCs expressed Sca-1. Indeed, $65 \%$ of the NMC population were positive for Sca-1 expression (Figure 1D). However, because several cell types were known to express Sca-1, we next isolated a Lin $^{-} \mathrm{NMC}$ population depleted of cells expressing CD45, CD31, CD34, Flk-1, CD3, CD4, CD8, B220, TER119, Mac-1, and $\mathrm{Gr}-1$. In this case, the fraction of Sca- $1^{+}$cells within the adult NMC population was drastically reduced to $33 \%$. Interestingly, the percentage of neonatal NMCs expressing Sca- 1 was not significantly changed following depletion $(11 \% \pm 3 \%$ vs. $7 \% \pm 1 \%$ in undepleted and depleted neonatal NMCs, respectively; Figure 1D).

In an attempt to expand a population of potential cardiac precursors, neonatal NMCs were cultured in vitro. After 2 passages, the expression of lineage-specific markers was analyzed by RT-PCR, immunohistochemistry, and flow cytometry (Figure 2). WT neonatal NMCs did not show significant expression of endothelial or hematopoietic cell markers, including CD31, Flk-1, CD45, CD34, TER119, Mac-1, Gr-1, and CD3 (Figure 2A). In addition, these cells did not express cardiac-specific proteins such as Nkx2.5, GATA-4, myosin light chain-2v (MLC-2v), troponin I, and cardiac actin (Figure 2B). In contrast, expanded NMCs expressed Sca-1, c-kit, and the transcription factors islet- 1 and Snail, as well as E-, VE-, and $\mathrm{N}$-cadherins (Figure 2, B and C). The number of Sca- $1^{+} \mathrm{NMCs}$ appeared to increase significantly during the expansion in culture (compare the percentage of neonatal Sca- $1^{+} \mathrm{NMCs}$ in Figure 1D, $11 \% \pm 3 \%$, with that in Figure $2 \mathrm{~A}, 30 \% \pm 6 \%$ ), whereas the fraction of cells expressing c-kit did not seem to change. Sca- $1^{+}$troponin ${ }^{-}$cells were small and presented a round shape that differed from that of cardiomyocytes (see Supplemental Figure 1; supplemental material available online with this article; doi:10.1172/JCI23418DS1). Interestingly, the percentages of adult NMCs expressing Sca-1 and c-kit following expansion in vitro (Figure 2A) were dramatically smaller than that observed immediately after isolation (Figure 1D) or in the corresponding neonatal population (Figure 2A).

Since neonatal NMCs demonstrated a capacity to be expanded in vitro as compared with adult NMCs, we assessed the capacity of these cells to differentiate into functional cardiomyocytes. 
A
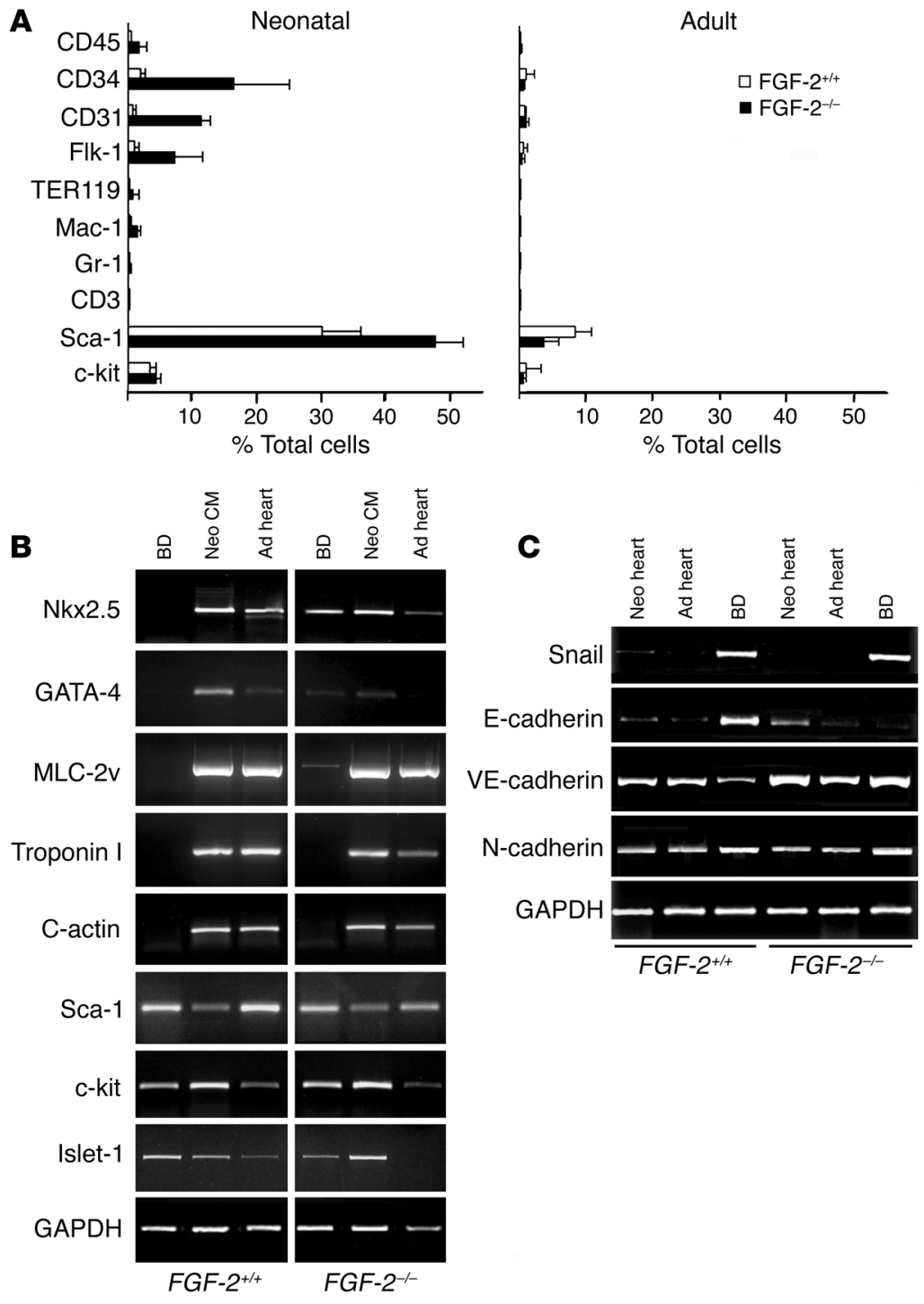

Figure 2

Characterization of the NMC population before differentiation. (A) Quantification by FACS analysis of neonatal and adult NMCs from FGF-2+/+ (white bars) and FGF-2 ${ }^{-/-}$mice (black bars) expressing CD31, Flk-1, CD45, CD34, TER119, Mac-1, Gr-1, CD3, Sca-1, and c-kit after expansion in vitro $(n=5-12)$. (B) RT-PCR analysis of cardiac-specific gene expression as well as Sca-1, c-kit, and islet-1 in NMCs after expansion and before differentiation (BD), in neonatal cardiomyocytes (Neo CM) and in the adult heart (Ad heart) from either $\mathrm{FGF}_{-2^{+/+}}$or $\mathrm{FGF}-2^{-/-}$mice. C-actin, cardiac actin. (C) RT-PCR analysis of Snail and cadherin expression in expanded NMCs (BD), as well as in the neonatal (Neo heart) and adult hearts (Ad heart) from $\mathrm{FGF}^{-2^{+/+}}$or $\mathrm{FGF}-2^{-/-}$mice.

after placement of expanded NMCs in differentiation medium (Figure 4A). Three weeks after the initiation of differentiation, approximately $5 \%$ of the cells in the culture showed a phenotype of mature cardiomyocytes (Figure 4B). In addition, spontaneous contractions were easily detected in the culture as early as 3 days after placement of the cells in differentiation medium. Interestingly, the differentiating cells often grew in clusters and demonstrated synchronized beatings. In parallel experiments, we evaluated the requirement for $c$-kit signaling during the differentiation of NMCs into cardiomyocytes using precursor cells isolated from mice with reduced c-kit signaling $\left(W / W^{v}\right.$ mutants; refs. 33, 34). Interestingly, full activation of c-kit was not mandatory for the cardiac precursor population to produce functional cardiomyocytes, since NMCs from mutant mice gave rise to spontaneously beating cells expressing cardiac markers (Supplemental Figure 3).

We also evaluated the multipotent capacity of the NMC precursors. Our attempts to clone these precursors were unsuccessful. Therefore, A variety of media were tested for the ability to sustain cardiogenic differentiation as judged by the appearance of cells demonstrating sarcomeric rearrangements (Supplemental Figure 2), and also spontaneous beatings. The medium referred to as differentiation medium in this article was the most potent in this assay (data not shown). Therefore, we determined whether precursor cells giving rise to functional cardiomyocytes expressed Sca-1. For this purpose, we followed the fate of Sca- $1^{+}$cells during the differentiation process. Figure 3, A-L, depicts the different cell types that were detected in the culture. During the first days, Sca- $1^{+}$ cells were essentially troponin ${ }^{-}$(Figure $3, \mathrm{~A}-\mathrm{C}$ ). Once again, at this point, the morphology of Sca- $1^{+}$troponin ${ }^{-}$cells differed markedly from that of cardiomyocytes. At a later stage, double-positive cells, i.e., Sca- $1^{+}$troponin ${ }^{+}$cells, represented the majority of the cells, although some cells were still single-positive for Sca-1 (Figure 3, D-F). Lastly, mature cardiomyocytes, which expressed only cardiac markers, were detected in more advanced cultures (Figure 3, G-I). Moreover, the expression in differentiating cells of early and late cardiac markers such as Nkx2.5, GATA-4, MEF-2c, MLC-2a, MLC-2v, troponin I, and cardiac actin was evident at 1 and 3 weeks the whole NMC population after expansion in vitro was plated in various media that were selected for their ability to sustain the differentiation into specific cell types. Under the appropriate conditions, NMCs produced adipocytes, skeletal myocytes, and osteoblasts, which were detected in the different cultures using specific staining and amplification of defined molecular markers (Figure 5). Finally, it is noteworthy that the presence of progenitors was also detected in NMC cultures based on Snail and islet-1 expression (Figure 2C).

Cardiogenic differentiation of cardiac progenitors is dependent on FGF-2. The capacity of cardiac precursors to be recruited for cardiac repair and to differentiate into fully functional myocytes depends on factors provided by the surrounding tissues. After expansion in vitro and before differentiation, the expression of FGF-2, FGF-1, bone morphogenetic protein-2 (BMP-2), BMP-4, TGF- $\beta 1$, and TGF- $\beta 2$ was easily detected in cardiac NMCs (Figure 6). FGF-4 and FGF-10 expression was barely detected, whereas FGF-8 did not seem to be expressed. Moreover, FGF-2 expression was absent in control undifferentiated culture as compared with what was seen in differentiation medium, in which FGF-2 and its cognate receptor (FGFR1) appeared 


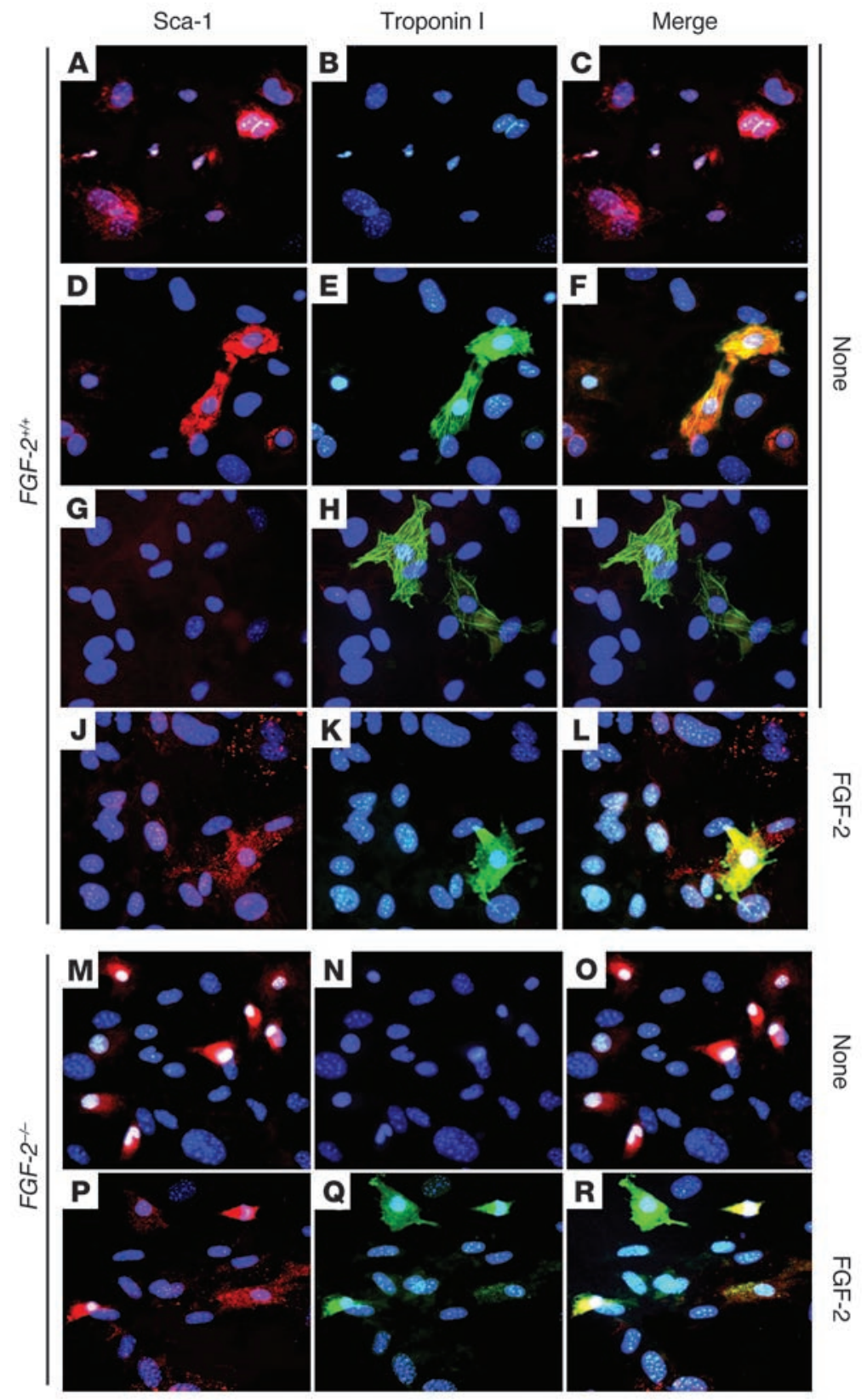

\section{Figure 3}

Cardiogenic differentiation of Sca- $1^{+}$cells in vitro. (A-L) Immunodetection of Sca-1 and troponin I in differentiating NMCs isolated from $\mathrm{FGF}-2^{+/+}$mice in the absence (None) or presence of FGF-2 and maintained for 1 (A-C), 2 (D-F), or 3 weeks (G-I) in differentiation medium. (M-R) The same experiment using NMCs isolated from FGF-2-/mice maintained for 3 weeks in differentiation medium. Original magnification, $\times 40$.

Next, the importance of FGF-2 in the differentiation capacity of cardiac precursors was evaluated in vitro using NMCs isolated from the neonatal heart of mice lacking FGF-2 expression. Immediately following isolation, the number of Sca- $1^{+}$cells in the cardiac myocyte and nonmyocyte fractions of neonatal FGF-2 knockouts was similar to that found in WT animals (Figure 1C). After expansion in vitro, Sca- $1^{+}$cells were also found in NMCs from FGF-2 knockouts (Supplemental Figure 1). The nonmyocyte population lacking FGF-2 demonstrated even a greater percentage of $\mathrm{Sca}-1^{+}$cells than that observed in WT controls $(30 \% \pm 6 \%$ vs. $48 \% \pm 5 \%$ in WTs and FGF-2 knockouts, respectively) (Figure 2A). Contrary to what we observed in WT NMCs, Nkx2.5 expression was detected in the FGF-2-deficient nonmyocyte fraction before induction of differentiation (Figure $2 \mathrm{~B}$ ). Interestingly, this population contained also a significant proportion of cells expressing CD34, Flk-1, and CD31 $(17 \% \pm 3 \%, 7 \% \pm 4 \%$, and $11 \% \pm 2 \%$, respectively; Figure $2 \mathrm{~A})$.

The capacity of FGF-2-deficient cells to produce cardiac myocytes was then tested. As shown in Figures 3 and 4, these cells, and specifically Sca- $1^{+}$ cells, failed to develop into functional cardiomyocytes, and no spontaneously contracting cells were observed in the culture. Accordingly, after 3 weeks in differentiation medium, expression of late cardiac markers in cells lacking FGF-2 was essentially absent (Figure 3, M-O, and Figure 4A). To evaluate whether the NMC population in FGF-2 knockouts was devoid of cardiac precursors or whether these precursors were stopped in their cardiogenic differentiation pathway because of a lack of signals,

largely upregulated. We therefore initiated a series of experiments aimed at assessing the role of FGF-2 in the maintenance, the recruitment, and the differentiation of cardiac precursors.

First, the presence of Sca- $1^{+}$cells in the heart of neonatal and adult FGF-2 knockouts was investigated. In neonates, cardiac Sca-1 expression did not differ from that of WTs (Figure 1, A and C). In contrast, Sca-1 staining in the heart of adult mice lacking FGF-2 was weaker than that observed in normal littermates (Figure 1A). Nevertheless, Sca-1 expression in the heart was upregulated in hypertensive mice with cardiac hemodynamic overload. Moreover, a significant percentage of adult NMCs lacking FGF-2 expressed Sca-1. However, the majority of these cells coexpressed lineage-specific markers (CD45, CD31, CD34, Flk-1, CD3, CD4, CD8, B220, TER119, Mac-1, and Gr-1) as shown by the decrease in percentage following depletion of lineage-committed cells (Figure 1D). exogenous FGF-2 was added to the culture (Figure 3, P-R, and Figure 4C). The addition of FGF-2 to cultures of NMCs lacking FGF-2 induced the reappearance of cells that express markers of mature cardiac myocytes (Figure 3, P-R, and Figure 4C). Moreover, in this case, cells expressing both Sca-1 and troponin I were readily detected in the culture (Figure 3, P-R), and spontaneously beating cells were also evident in this case. In control experiments, we evaluated the potential of FGF-1 to support the production of cardiomyocytes by FGF-2-deficient NMCs. In these experiments, FGF-1 was unable to replace FGF-2 (Figure 4C). Finally, the possibility that the absence of FGF-2 resulted in an increased susceptibility of FGF-2-deficient NMCs to apoptosis was investigated. NMCs from neonatal FGF-2 knockouts, maintained in control or differentiation medium, demonstrated a small number of apoptotic cells, which was similar to that 
A

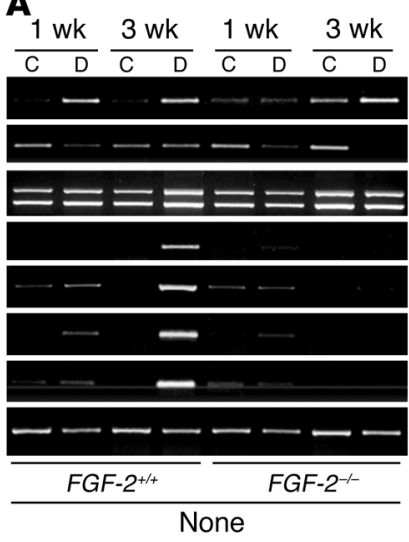

Nkx2.5

GATA-4

MEF-2c

MLC-2a

MLC-2v

Troponin I

C-actin

GAPDH

C

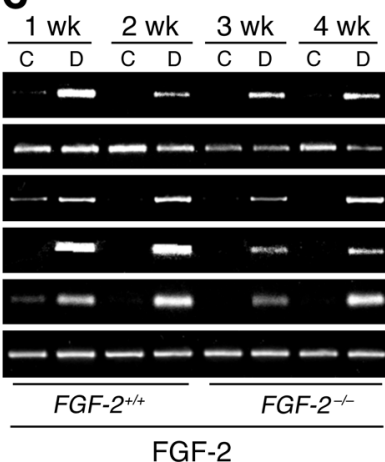

FGF-2
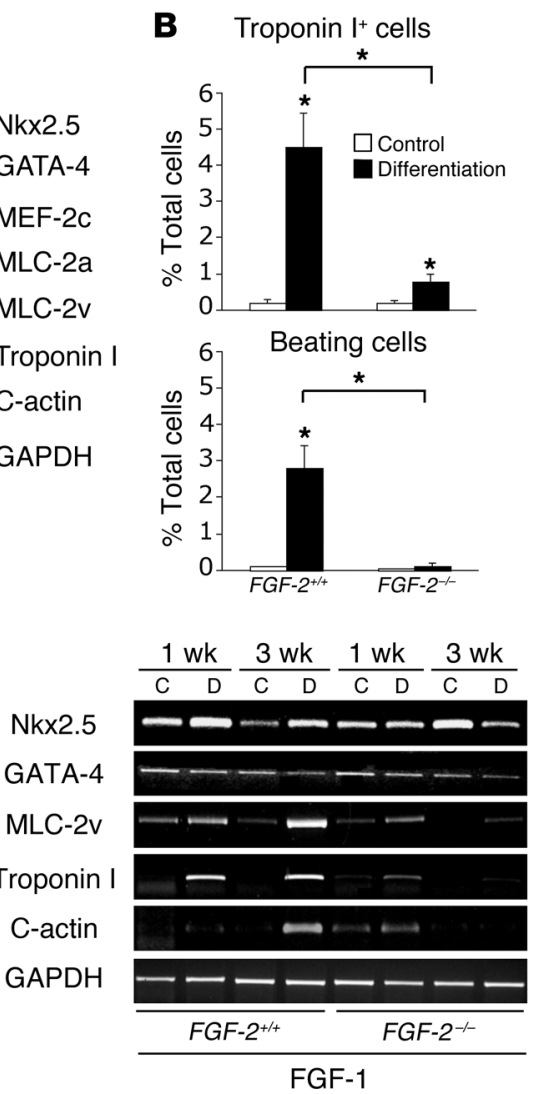

Figure 4

Differentiation of cardiac precursors into functional cardiomyocytes. (A) RT-PCR analysis of cardiac-specific gene expression in NMCs from $\mathrm{FGF}-2^{+/+}$or $\mathrm{FGF}-2^{-/-}$mice maintained in control (C) or differentiation medium (D) in the absence of FGF-2 (None), after 1 week or 3 weeks in culture. (B) Quantification of troponin $\mathrm{I}^{+}$cells and spontaneously contracting cells in culture of differentiated cardiac precursor from $F G F-2^{+/+}$ and $F G F-2^{-/-}$mice after 3 weeks in control medium (white bars) or differentiation medium (black bars); $n=6-10$. ${ }^{\star} P<0.05$. (C) RT-PCR analysis of cardiac-specific gene expression in NMCs from $\mathrm{FGF}-2^{+/+}$or $\mathrm{FGF}_{-2} \mathrm{2}^{-/}$mice maintained in control or differentiation medium in the presence of FGF-2 or FGF-1 after 1 week or 3 weeks in culture.

$\alpha$-actinin. Some fluorescent cells were also detected in the liver and in skeletal muscles. However, the injected cells did not differentiate into the respective tissue (data not shown). Interestingly, fluorescence-activated cell sorting-selected (FACS-selected) $\mathrm{Sca}-1^{+}$cells had a greater potential for engraftment as compared with Sca- $1^{-}$cells (percentages of successful transfers were $100 \%$ vs. $40 \%$ in recipients having received FACS-purified Sca- $1^{+}$and Sca- ${ }^{-}$ cells, respectively; $n=4-5$ mice per group).

We next performed a series of experiments aimed at assessing the need for FGF-2 in the engraftment and differentiation processes in vivo. Therefore, WT NMCs as well as NMCs lacking FGF-2 expression were injected into either WT or FGF-2-deficient neonates (Figure 7B). Homing was observed in all groups regardless of the capacity observed in WT cultures (Supplemental Figure 4). Specifically, percentages of apoptotic cells in UV-irradiated cultured cells (positive control), in cells maintained in control medium, and in cells maintained in differentiation medium were $10.5 \% \pm 2.5 \%$, $2.3 \% \pm 1.2 \%$, and $3.2 \% \pm 1.5 \%$, respectively, for WT NMCs, and $11.3 \% \pm 1.5 \%, 2.7 \% \pm 0.9 \%$, and $2.9 \% \pm 1.2 \%$, respectively, for FGF-2-deficient NMCs.

Homing capacity of cardiac precursors in vivo. To evaluate the ability of cardiac precursors to home to the heart, we performed cell transfer experiments. In a first series of experiments, the cells were injected into neonatal mice to evaluate their potential to contribute to the physiological postnatal remodeling of the heart. Therefore, WT NMCs were labeled with the membrane dye PKH2-GL, and infused i.v. into recipient mice. In parallel experiments, NMCs expressing a GFP transgene under control of the chicken $\beta$-actin promoter were used. Figure 7A demonstrates that the cells were found in cardiac tissues 10 days after infusion, and that these cells presented characteristics of differentiated cardiomyocytes. In particular, PKH2-GL-labeled and GFP-expressing cells expressed

\section{Figure 5}

Multipotent capacity of cardiac precursors. (A) Oil red staining and RT-PCR analysis of PPAR $\gamma$ and lipoprotein lipase (LPL) expression in cultures of NMCs induced to differentiate into adipocytes. (B) Immunodetection of MyoD1 and RT-PCR analysis of MyoD1 expression in cultures of NMCs induced to differentiate into skeletal myoblasts. (C) Immunodetection of osteocalcin and RT-PCR analysis of osteocalcin expression in cultures of NMCs induced to differentiate into osteoblasts. Original magnification, $\times 20(\mathbf{A}$ and $\mathbf{C})$, $\times 30(C$, inset) $, \times 40(B), \times 63$ (B, inset). of the recipients or the injected cells to synthesize FGF-2. However, differentiation in situ was only seen if either the recipient animal or the injected cells were able to produce FGF-2. Indeed, no differentiation was observed in FGF-2-deficient mice receiving NMCs lacking FGF-2 expression. The success rate of homing
A

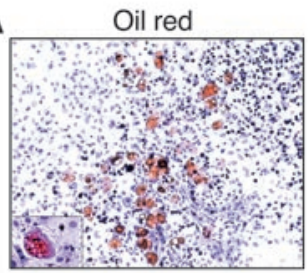

B

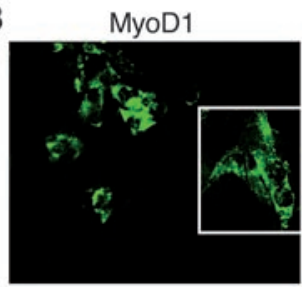

C

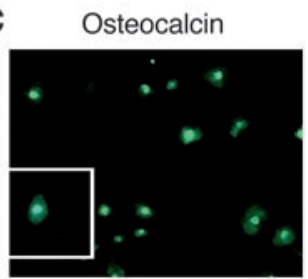

BD $C \quad D$

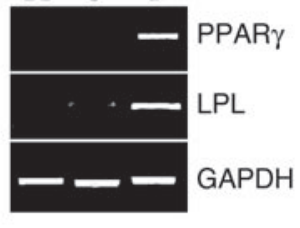

BD C D

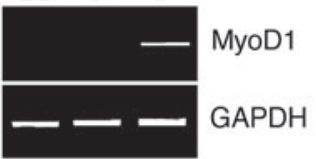

BD

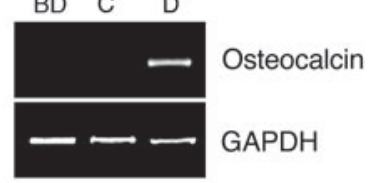




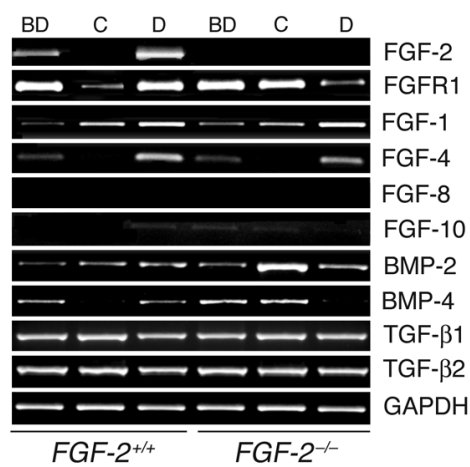

Figure 6

Factor expression in NMCs. RT-PCR analysis of factor expression in NMCs from $\mathrm{FGF}_{-2} \mathrm{2}^{+/+}$and $\mathrm{FGF}-2^{-1-}$ mice after expansion in vitro (before differentiation) as well as after 3 weeks in control and differentiation medium.

following NMC injections was also reduced in this latter case. Specifically, whereas $100 \%$ of the transferred animals demonstrated engraftment when FGF-2 was produced by either the recipient or the injected cells, only $40 \%$ of FGF- 2 knockouts having received NMCs lacking FGF-2 demonstrated significant homing $(n=9-12)$. Finally, to exclude that cardiac precursors contribute to cardiac morphogenesis via cell fusion, we performed a series of control experiments, in which PKH2-GL-labeled cells were infused into ROSA26 neonatal mice constitutively expressing the LacZ gene encoding $\beta$-gal. We then searched cardiac tissues for PKH2-GLlabeled cells that would also express the LacZ transgene but could not find any dually stained cells (Figure 7C).

Then, neonatal NMCs were administrated to adult mice. Two weeks after injection, PKH2-GL-labeled cells were identified in the cardiac muscle (Figure 8A). These cells demonstrated a morphology corresponding to that of cardiomyocytes and expressed troponin I (Figure 8B). Cardiac precursors from ROSA26 transgenics were also infused in WT and FGF-2-deficient mice. These cells were readily identified in cardiac tissues by immunostaining as late as 4 weeks after injection (Figure 8C), and the expression of LacZ transgene was also detected by PCR (Figure 8D).

\section{Discussion}

In the present study, we demonstrate that the cardiac NMC population contains a subset of cells that express the Sca-1 antigen as well as mesenchymal markers and have the capacity to differentiate into functional cardiomyocytes. Because these cells do not express CD45, CD34, and c-kit, they are reminiscent of skeletal myogenic precursors (35). These precursors can home to the heart after injection into immunocompetent recipient mice and contribute to cardiac tissues. Interestingly, the differentiation process depends on FGF-2, since cells lacking FGF-2 failed to produce mature cardiomyocytes and stop their differentiation at a precursor stage.

Cell-based therapies to replace lost tissues in the damaged heart suggest a promising approach for the treatment of cardiac diseases. Different cell types have been assayed for their capacity to differentiate into cardiac myocytes. For instance, spontaneously contracting cardiomyocytes can easily be produced from ES cells in vitro (36). These cells can even colonize the adult heart and differentiate into contractile tissues $(37,38)$. However, ES cell-derived cardiomyocytes resemble cardiac myocytes of the primary heart tube (39), and the risk of uncontrolled proliferation of teratoma cells, as well as ethical reasons, is likely to reduce their wide use in therapies $(40,41)$. Adult HSCs, as well as stromal stem cells isolated from the bone marrow and endothelial progenitor cells, turn into cardiomyocytes under the appropriate culture conditions (6-9). Bone marrow cells have even been claimed to differentiate into cardiac myocytes in the infarcted area of the heart, where they were suggested to contribute to myocardium regeneration (8). However, this view has been recently challenged, and it is therefore not clear whether bone marrow cells represent a valuable source of precursors for cardiac repair (12-14).

Our data demonstrate that the neonatal heart contains a population of undifferentiated precursors that can be induced in vitro to give rise to functional mature cardiomyocytes. These cells are found in the nonmyocyte population of the heart, which includes cells derived from the cardiac mesenchyme that result from the epithelial-to-mesenchymal transformations (EMTs) taking place during heart development (42-44). Transforming cells express the transcription factor Snail, which has been suggested to represent a marker of epithelial-derived mesenchymal cells in the developing heart $(43,45)$. Adoption of a migrative phenotype requires the downregulation of adhesion molecules, including cadherins. Interestingly, Snail expression appears to be under control of tyrosine kinase receptors, including FGFR1, and therefore of FGF-2 $(44,46)$. Indeed, cardiac NMCs in the present study concomitantly expressed FGF-2, FGFR1, Snail, and cadherins. Nevertheless, culture of NMCs lacking FGF-2 expressed Snail at similar levels to those found in WT cells (Figure 2C). It is therefore likely that certain redundant pathways, which might involve other FGF family members, control Snail expression. Furthermore, EMT in the heart requires the presence of TGF- $\beta 1$ and TGF- $\beta 2$ (47), which are also expressed by cardiac NMCs. During cardiac morphogenesis, 1 EMT occurs during endocardial cushion formation and cellularization of the cardiac jelly. Interestingly, a second EMT has been suggested to involve a subset of epicardial cells that can migrate into the myocardium (43). These cells can subsequently differentiate in vitro and in vivo into several cell types, including cardiac fibroblasts and cardiomyocytes.

In addition, cardiac NMCs expressed islet- 1 after expansion in vitro. This transcription factor has been shown to identify cardiac progenitors in the developing heart $(48,49)$. It is noteworthy that islet- 1 expression is downregulated in differentiating cells, and islet- 1 could be therefore important in maintaining an undifferentiated state (49). Interestingly, islet- $1^{+}$cardioblasts with a capacity to differentiate into mature cardiomyocytes were recently demonstrated to be present in the nonmyocyte fraction of the neonatal mouse heart (50). In addition, since adult FGF-2 knockouts do not demonstrate cardiac islet- 1 expression, this could suggest that the heart of adult mice lacking FGF-2 expression is devoid of a particular cardiac precursor subset. Our data suggest also that precursors in the cardiac NMC population demonstrate mesenchymal characteristics, since they appear to be able to produce adipocytes, osteoblasts, and skeletal muscle cells in addition to functional cardiomyocytes. Nevertheless, one cannot exclude at this point that these different cell types arise from distinct precursors present in the cardiac NMC population.

A significant fraction of cardiac progenitors express Sca-1. The percentage of Sca- $1^{+}$cells in the neonatal NMC population increased during the expansion in vitro. At this point, it is, however, difficult to evaluate whether this increase results from proliferation of Sca- $1^{+}$cells or the conversion of Sca- $1^{-}$cells. Nevertheless, the 
A

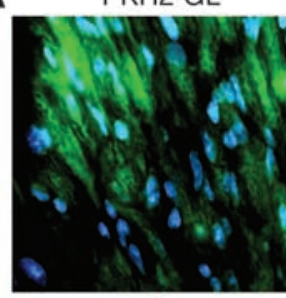

GFP

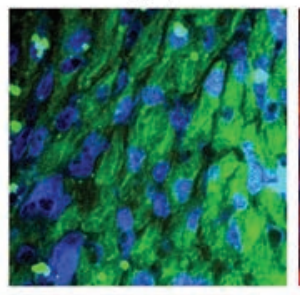

B
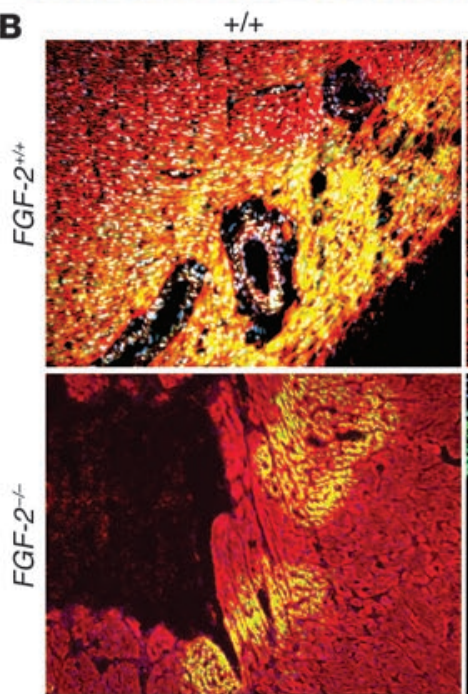

C

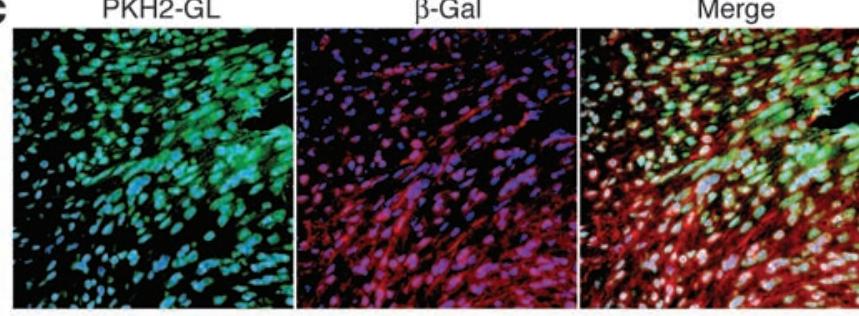

$\alpha$-Actinin

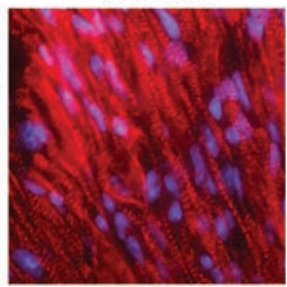

$\alpha$-Actinin
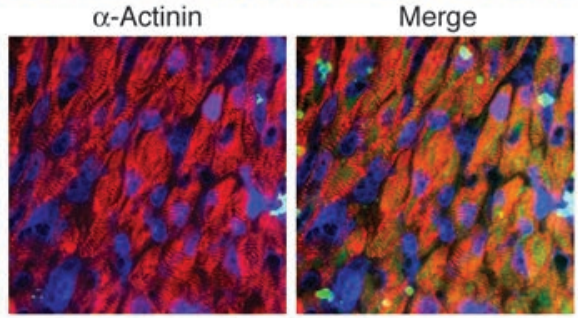

$-1-$
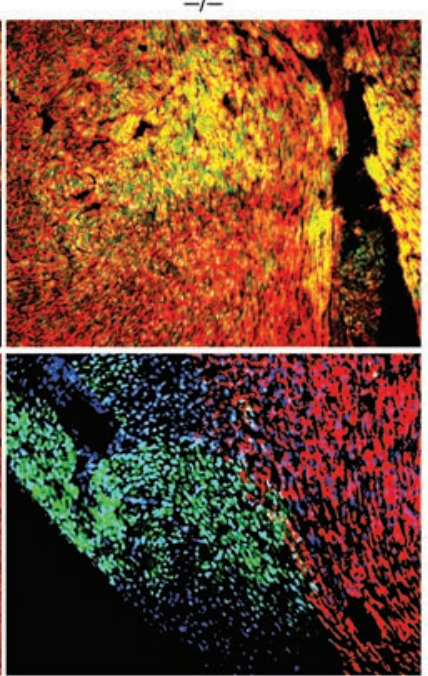

Merge

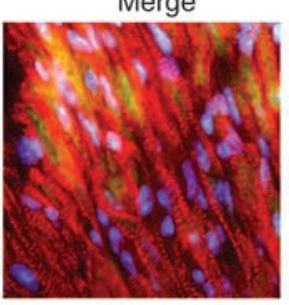

Merge

\section{(1)}

\section{Figure 7}

Cell transfer into neonatal mouse recipients. (A) PKH2-GL-labeled cells or cells constitutively expressing the GFP gene under the chicken $\beta$-actin promoter (GFP) were injected i.v. into neonatal mice. Ten days thereafter, heart sections were stained for $\alpha$-actinin. (B) PKH2-GL-labeled $F G F-2^{+/+}$or $F G F-2^{-1-}$ NMCs were injected into neonatal $F G F-2^{+/+}$or $F G F-2^{-/-}$mice. Ten days after injection, heart sections were stained for $\alpha$-actinin. (C) WT PKH2GL-labeled NMCs were injected into neonatal ROSA26 transgenic mice, and heart sections were stained for $\beta$-gal. Original magnification, $\times 63(\mathbf{A}$ and $\mathbf{C}), \times 20(\mathbf{B})$.

were recruited to the damaged area and participated in the healing process. These progenitors differ somehow from the cells described herein in their expression of particular cell surface markers. In 1 study (54), cardiac progenitors were isolated from the adult heart based on Sca-1 expression. Interestingly, a significant portion of these cardiac progenitors appeared to express high levels of CD31 and CD38, suggesting that they might derive from endothelial precursors. In contrast, CD31 expression is absent on the surface of the WT NMCs described in the present study. Then, a $\mathrm{Lin}^{-}, \mathrm{c}-\mathrm{kit}^{+}$cell population was identified in the adult heart as containing cardiac precursors (55). These cells, or their clonal progeny, reconstitute a differentiated myocardium following injection into the border of an infarcted ventricular area. Along these lines, the isolation of cardiac stem cells from human atrial and ventricular biopsies as well as from the adult mouse heart has been reported (56). These undifferentiated precursors display self renewal and express, for the most part, the c-kit antigen. Only a small fraction of the NMC population in our study appeared to express $c$-kit. In addition, NMCs isolated from $W / W^{v}$ mice demonstrate cardiogenic conversion. Interestingly, hearts from $W / W^{v}$ mice were reported to demonstrate a greater capacity to adapt to pressure overload than their WT counterparts (57). Indeed, whereas ventricular performance decreased gradually in aortic-banded WT mice, decompensation did not occur in $W / W^{v}$ mice. Therefore markers such as Sca-1, c-kit, and islet-1 appear to be expressed by cardiac precursors under different conditions. At present, it is possible that all these different cell subsets contribute to maintain precursor pools in the heart and might even constitute together the so-called stem cell niches within the differentiated tissue.

The differentiation of cardiac precursors into mature cardiomyocytes appears to be dependent on the capacity of the cells to produce FGF-2. The lack of FGF-2 expression does not

Sca- $1^{+}$cells can produce a cardiogenic progeny under the appropriate conditions. Interestingly, $\mathrm{Sca}-1^{+}$cells are also found in the adult heart, and cardiac expression of the Sca- 1 antigen is increased during hemodynamic overload. However, in our hands, the Sca- $1^{+}$ NMC population from adult mice appeared to present a lesser degree of proliferative capacity in vitro than that from neonates. Mice lacking Sca-1 expression have been recently described (51). Although these animals are viable and grossly normal under basal conditions, they suffer from a defect in mesenchymal stem cell self renewal, which could be responsible for age-related pathologies (52, 53). Therefore, it is proposed that Sca-1 could play a role in modifying the balance between self renewal and differentiation.

Recent studies reported the existence of Sca- $1^{+}$cardiac precursors in the adult heart $(54,55)$. Upon cardiac injury, these cells

affect the number of precursors in the heart, since the differentiation process can be restored in vitro by the addition of exogenous FGF-2, and apoptosis did not appear to be increased in the FGF-2deficient population. On the contrary, Sca- $1^{+}$cells accumulate in the culture in the absence of FGF-2. Interestingly, some progenitor cells have been shown to express FGFR1 and to be dependent on FGF-2 for their expansion (30, 31). Cardiac Sca-1 expression in FGF-2 knockouts is revealed during hemodynamic stress (Figure $1 \mathrm{~A})$. In the bone marrow, mesenchymal stem cells, referred to also as multipotent adult progenitor cells, express low levels of Sca-1 (58). It is therefore possible that, in vivo, in the absence of FGF-2, progenitor cells accumulate at an early stage that does not express Sca-1. In contrast, after expansion in vitro, a Sca- $1^{+}$population accumulates in NMCs lacking FGF-2 expression (Figure 2A). 


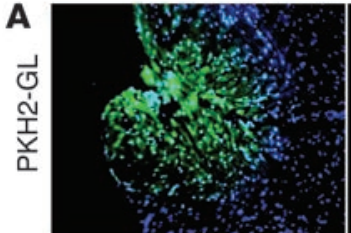

$\mathbf{B}$

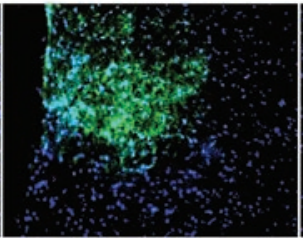

Troponin I

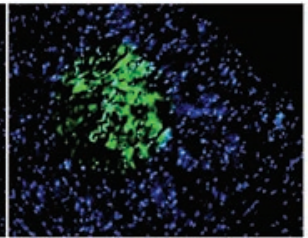

Merge

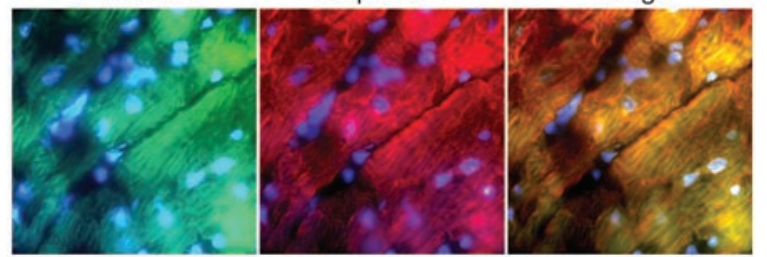

C

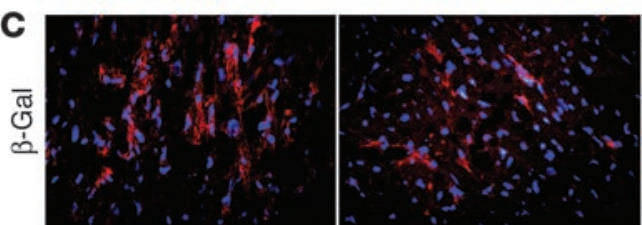

D

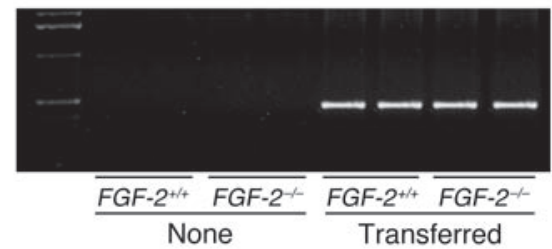

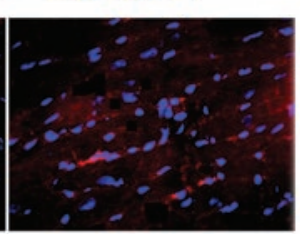

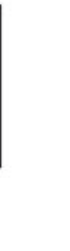

However, a significant percentage of precursor cells lacking FGF-2 express CD31, CD34, and Flk-1. This would suggest the accumulation in the culture of cells with a phenotype corresponding to the hemangioblast precursor (29).

In any case, these data indicate that the failure to produce FGF-2 prevents cardiac precursors from completing a normal cardiogenic differentiation. This is confirmed in experiments performed in vivo, in which differentiation is abolished in the absence of FGF-2 expression in both the transferred population and the recipient animals. Different members of the FGF family, together with other proteins such as TGFs or BMPs, have been implicated in cardiogenesis $(28,59)$. In particular, FGF-2 appears to be required for the expression of cardiac transcription factors and the differentiation of mesoderm explants induced by BMP-2. Moreover, FGF-2 induces mesenchymal cell formation from precardiac mesoderm explants and was shown to stimulate EMT in the epicardium for the production of epicardial precursor cells $(27,42,43,60)$. As mentioned above, epicardially derived cells were suggested to represent a source of cardiac stem cells. Nevertheless, mice deficient in FGF-2 expression do not suffer from major heart defects during embryonic life $(21,61)$. This suggests that other members of the FGF family compensate for the lack of FGF-2 expression in the embryo, for instance FGF-4 or FGF-8 (59). This would also indicate that FGF-2 is more important for maintaining cardiac integrity after birth. Alternatively, one cannot exclude that FGF-2 acts preferentially on a particular subset of cardiac precursors, which, according to our data, would express Sca-1. Interestingly, FGF-2deficient mice used in the present study suffer from an impaired hypertrophic response and dilated cardiomyopathy (21). Although the dilated phenotype was not always observed when independent FGF-2 knockouts were used (61), all models are characterized by

\section{Figure 8}

Cell transfer into adult mouse recipients. (A) PKH2-GL-labeled cells isolated from WT hearts were injected into adult FGF-2+/+ mice. Original magnification. (B) Troponin I expression of differentiated precursors in WT recipient hearts. Two weeks after cell transfer, heart sections were stained for $\alpha$-actinin. (C) NMCs isolated from ROSA26 transgenic mice were injected into adult $\mathrm{FGF}-2^{+/+}$mice. Sections were immunostained for $\beta$-gal 4 weeks after transfer. (D) RT-PCR analysis of $L a c Z$ gene expression in hearts from $\mathrm{FGF}-2^{+/+}$and $F G F-2^{-/-}$mice after transfer of NMCs from ROSA26 transgenic mice (Transferred). None, no cells transferred. Magnification, $\times 20(\mathbf{A}), \times 100(B), \times 40(C)$.

an absence of cardiac remodeling in response to pressure overload. Our data provide therefore an attractive hypothesis for explaining this defective cellular response. Finally, other factors are likely to play a role in the recruitment, survival, and differentiation of cardiac precursors. For instance, IGF-1 was recently shown to prevent myocardial dysfunction via the preservation of a pool of undifferentiated precursors, which could be mobilized to compensate for cardiac cell death (62).

Together, our data provide further evidence that the heart contains a population of undifferentiated precursors that could be recruited for the production of functional cardiomyocytes. These cardiac precursors are probably derived from a mesodermal population with characteristics of mesenchymal and/or hemangioblast precursors. Importantly, the differentiation process requires the presence of FGF-2. Several clinical trials have been launched to evaluate the therapeutic potential of FGF-2 in cardiac diseases (22-25). In particular, FGF-2 is currently tested as a drug capable of stimulating revascularization for the treatment of chronic ischemic conditions. Our results suggest that the beneficial effects of FGF- 2 could extend beyond its angiogenic actions. Indeed, FGF-2 could participate in the self regeneration of the heart by accelerating the mobilization and differentiation of resident stem cells for cardiac repair.

\section{Methods}

Mice. Neonatal C57BL/6 mice (Charles River Laboratories) were used as a source of NMCs. Adult and neonatal mice lacking FGF-2 (21) maintained on a $\mathrm{C} 57 \mathrm{BL} / 6$ background, transgenic mice bearing the LacZ reporter gene [129S7-Gt(ROSA)26Sor; Jackson Laboratory] or the chicken $\beta$-actin-GFP reporter (63), and $W / W^{v}$ mice (deficient in c-kit signaling) $(33,34)$ were used in some experiments. Animals were handled according to institutional guidelines after approval from the State Veterinary Office (Lausanne, Switzerland).

Cell culture and cardiogenic differentiation. Cardiac NMCs were isolated as previously described (21). Briefly, ventricles were digested in buffer containing $0.45 \mathrm{mg} / \mathrm{ml}$ collagenase (Worthington Biochemical Corp.) and $1 \mathrm{mg} / \mathrm{ml}$ pancreatin (Invitrogen Corp.). Cardiomyocytes and NMCs were separated by differential plating (45 minutes), and expanded in culture in a 3:1 mixture of DMEM and Medium 199 (Invitrogen Corp.) supplemented with 10\% horse serum (Serotec), 5\% FBS (Serotec), $100 \mathrm{U} / \mathrm{ml}$ penicillin G, and $100 \mu \mathrm{g} / \mathrm{ml}$ streptomycin. After 2 passages, cells were switched to a differentiation medium composed of MEM Alpha (Invitrogen Corp.), 10\% FBS, and supplemented with $1 \mu \mathrm{M}$ dexamethasone (Sigma-Aldrich), $50 \mu \mathrm{g} / \mathrm{ml}$ ascorbic acid (SigmaAldrich), and $10 \mathrm{mM} \beta$-glycerophosphate (Sigma-Aldrich). Control medium was MEM plus FBS. In some cases, media were supplemented with $25 \mathrm{ng} / \mathrm{ml}$ FGF-2 (R\&D Systems) or FGF-1 (Upstate) for 4 or 10 days, respectively. 
Differentiation into other cell types. For inducing differentiation into adipocytes, osteoblasts, and skeletal myocytes, NMCs were incubated in the following media, respectively: MEM/FBS containing $1 \mu \mathrm{M}$ dexamethasone, $0.5 \mathrm{mM} 3$-isobutyl-1-methylxanthine, and $5 \mu \mathrm{g} / \mathrm{ml}$ insulin; MEM/FBS containing $1 \mu \mathrm{M}$ dexamethasone, $50 \mu \mathrm{g} / \mathrm{ml}$ ascorbic acid, $10 \mathrm{mM} \beta$-glycerophosphate, and $10 \mathrm{nM} 1-\alpha, 25$-dihydroxyvitamin $\mathrm{D}_{3} ; \mathrm{MEM} / \mathrm{FBS}$ containing $1.5 \mu \mathrm{g} / \mathrm{ml}$ amphotericin B.

RT-PCR. Total RNA was extracted from either cultured cells or cardiac tissues (RNeasy Mini Kit; QIAGEN), reverse-transcribed $\left(30\right.$ minutes, $\left.55^{\circ} \mathrm{C}\right)$, and amplified by PCR (OneStep RT-PCR kit; QIAGEN). Primers and optimal annealing temperatures are listed in Supplemental Table 1. PCR products were run on a $2 \% \mathrm{NuSieve}$ GTG agarose gel (Cambrex Corp.).

Immunohistochemistry. For immunostaining, NMCs were fixed with $4 \%$ paraformaldehyde in PBS, and permeabilized with $0.2 \%$ Triton X-100 in PBS. Cells were incubated with antibodies directed to sarcomeric $\alpha$-actinin (Sigma-Aldrich), osteocalcin (ANAWA Trading SA), Sca-1 (BD Biosciences), troponin I-C, vWF (factor VIII), and MyoD1 (all from Santa Cruz Biotechnology Inc.). Fluorophore-conjugated secondary antibodies were as follows: FITC-labeled anti-mouse IgG, anti-rabbit IgG, and anti-goat IgG (all from Sigma-Aldrich). Antibodies were diluted in PBS containing $1 \%$ nonimmune goat serum (Sigma-Aldrich) or BSA (Sigma-Aldrich). DNA was stained with DAPI (Invitrogen Corp.). Heart cryosections from neonatal and adult mice were fixed with $4 \%$ paraformaldehyde and stained with either a rat anti-Sca-1 (BD Biosciences), a mouse anti- $\alpha$-actinin (Sigma-Aldrich), a mouse anti- $\beta$-gal (Sigma-Aldrich), or a goat anti-troponin I-C (Santa Cruz Biotechnology Inc.). The appropriate secondary antibodies were coupled with Alexa 488 or Alexa 594 fluorochrome (Invitrogen Corp.). Slides were mounted with FluorSave reagent (Merck Biosciences) and examined with a Carl Zeiss Axiophot I microscope equipped with an AxioCam color CCD camera (Carl Zeiss). For oil red staining, cells were washed 3 times with PBS and fixed for 4 minutes in 3.7\% formaldehyde. Oil red O (0.5\% in isopropanol; Sigma-Aldrich) was diluted 4 times in $\mathrm{H}_{2} \mathrm{O}$, and added to the cells for 1 hour at room temperature.

FACS. Cells were resuspended in PBS containing 3\% FBS and $2 \mathrm{mM}$ EDTA, and labeled with various antibodies for 30 minutes on ice. Antibodies used in this study were cyanine 5 (Cy5) anti-mouse Ly-6A/E (Sca-1) (eBioscience), FITC anti-CD45, PE anti-CD117, PE anti-c-kit, PE anti-CD34, PE anti-Flk-1, FITC anti-CD3, and FITC anti-B220. In some experiments, cells were incubated with rat antibodies directed against
Gr-1, Mac-1, CD31, and TER119 and a FITC-labeled anti-rat antibody of goat origin (all from BD Biosciences). In some experiments, suspensions were depleted of cells expressing CD45, CD31, CD34, Flk-1, CD3, CD4, CD8, B220, TER119, Mac-1, and Gr-1 following incubation with specific rat antibodies directed against mouse proteins and anti-rat magnetic beads (Dynabeads; Dynal Biotech).

Apoptosis assay. Cells were UV-irradiated (positive control), or maintained in either control or differentiation medium, and stained for 15 minutes with Hoechst $33342(10 \mu \mathrm{g} / \mathrm{ml})$. Then, the number of damaged nuclei was determined.

Cell transfer. Undifferentiated NMCs from WT, $\beta$-actin-GFP, or ROSA26 mice were isolated from neonatal hearts, and expanded in culture. WT NMCs were labeled with membrane dye PKH2-GL (Sigma-Aldrich; $4 \times 10^{-6} \mathrm{M}, 5$ minutes, room temperature). Cells $\left(10^{5}\right.$ in $100 \mu \mathrm{l} \mathrm{PBS}, 2 \mathrm{mM}$ EDTA) were injected into neonatal mice through the superficial temporal vein. Cells $\left(6 \times 10^{5}\right.$ in $600 \mu \mathrm{l}$ PBS, $2 \mathrm{mM}$ EDTA) were injected into adult mice via the tail vein. Cellular engraftment was determined 1 week or 2 weeks after transfer in neonatal mice and adult mice, respectively. Renovascular hypertension (2-kidney 1-clip model) was produced in adult mice as previously described (32).

Statistical analysis. One-way ANOVA was used to test for differences between groups. $P$ values of less than 0.05 were considered significant.

\section{Acknowledgments}

The expert technical assistance of Jean-Francois Aubert is gratefully acknowledged. We thank Anne Wilson for sharing her expertise in FACS analysis, Konstantinos Kaloulis for valuable discussions, and Christian Widmann for helping with the detection of apoptosis. This work was supported in part by a grant from the Leenaards Foundation (Lausanne, Switzerland) and by the Swiss National Science Foundation (TP grant 3200BO-102154).

Received for publication September 21, 2004, and accepted in revised form April 5, 2005.

Address correspondence to: Thierry Pedrazzini, Department of Medicine, University of Lausanne Medical School, CH-1011 Lausanne, Switzerland. Phone: 41-21-314-0765; Fax: 41-21-314-5978; E-mail: thierry.pedrazzini@hospvd.ch.
1. Lopez, A.D., and Murray, C.C. 1998. The global burden of disease, 1990-2020. Nat. Med. 4:1241-1243.

2. Mann, D.L. 1999. Mechanisms and models in heart failure: a combinatorial approach. Circulation. 100:999-1008.

3. Sussman, M.A., and Anversa, P. 2004. Myocardial aging and senescence: where have the stem cells gone? Annu. Rev. Physiol. 66:29-48.

4. Weissman, I.L., Anderson, D.J., and Gage, F. 2001 Stem and progenitor cells: origins, phenotypes, lineage commitments, and transdifferentiations. Annu. Rev. Cell Dev. Biol. 17:387-403.

5. Strauer, B.E., and Kornowski, R. 2003. Stem cell therapy in perspective. Circulation. 107:929-934.

6. Fukuda, K. 2001. Development of regenerative cardiomyocytes from mesenchymal stem cells for cardiovascular tissue engineering. Artif. Organs. 25:187-193.

7. Makino, S., et al. 1999. Cardiomyocytes can be generated from marrow stromal cells in vitro. J. Clin. Invest. 103:697-705.

8. Orlic, D., et al. 2001. Bone marrow cells regenerate infarcted myocardium. Nature. 410:701-705.

9. Badorff, C., et al. 2003. Transdifferentiation of blood-derived human adult endothelial progenitor cells into functionally active cardiomyocytes.

\section{Circulation. 107:1024-1032.}

10. Terada, N., et al. 2002. Bone marrow cells adopt the phenotype of other cells by spontaneous cell fusion. Nature. 416:542-545.

11. Ying, Q.L., Nichols, J., Evans, E.P., and Smith, A.G. 2002. Changing potency by spontaneous fusion. Nature. 416:545-548.

12. Murry, C.E., et al. 2004. Haematopoietic stem cells do not transdifferentiate into cardiac myocytes in myocardial infarcts. Nature. 428:664-668.

13. Balsam, L.B., et al. 2004. Haematopoietic stem cells adopt mature haematopoietic fates in ischaemic myocardium. Nature. 428:668-673.

14. Nygren, J.M., et al. 2004. Bone marrow-derived hematopoietic cells generate cardiomyocytes at a low frequency through cell fusion, but not transdifferentiation. Nat. Med. 10:494-501.

15. Fuchs, E., Tumbar, T., and Guasch, G. 2004. Socializing with the neighbors: stem cells and their niche. Cell. 116:769-778.

16. Szebenyi, G., and Fallon, J.F. 1999. Fibroblast growth factors as multifunctional signaling factors. Int. Rev. Cytol. 185:45-106.

17. Okada-Ban, M., Thiery, J.P., and Jouanneau, J. 2000. Fibroblast growth factor-2. Int. J. Biochem. Cell Biol. 32:263-267.
18. Detillieux, K.A., Sheikh, F., Kardami, E., and Cattini, P.A. 2003. Biological activities of fibroblast growth factor- 2 in the adult myocardium. Cardiovasc. Res. 57:8-19.

19. Jiang, Z.S., et al. 2002. Acute protection of ischemic heart by FGF-2: involvement of FGF-2 receptors and protein kinase C. Am. J. Physiol. Heart Circ. Physiol. 282:H1071-H1080.

20. Sheikh, F., Sontag, D.P., Fandrich, R.R., Kardami, E., and Cattini, P.A. 2001. Overexpression of FGF-2 increases cardiac myocyte viability after injury in isolated mouse hearts. Am. J. Physiol. Heart Circ. Physiol. 280:H1039-H1050.

21. Pellieux, C., et al. 2001. Dilated cardiomyopathy and impaired cardiac hypertrophic response to angiotensin II in mice lacking FGF-2. J. Clin. Invest. 108:1843-1851. doi:10.1172/JCI200113627.

22. Simons, M., and Ware, J.A. 2003. Therapeutic angiogenesis in cardiovascular disease. Nat. Rev. Drug Discov. 2:863-871.

23. Laham, R.J., et al. 1999. Local perivascular delivery of basic fibroblast growth factor in patients undergoing coronary bypass surgery: results of a phase I randomized, double-blind, placebo-controlled trial. Circulation. 100:1865-1871.

24. Simons, M., et al. 2002. Pharmacological treatment 
of coronary artery disease with recombinant fibroblast growth factor-2: double-blind, randomized, controlled clinical trial. Circulation. 105:788-793.

25. Ruel, M., et al. 2002. Long-term effects of surgical angiogenic therapy with fibroblast growth factor 2 protein. J. Thorac. Cardiovasc. Surg. 124:28-34.

26. Sugi, Y., Sasse, J., and Lough, J. 1993. Inhibition of precardiac mesoderm cell proliferation by antisense oligodeoxynucleotide complementary to fibroblast growth factor-2 (FGF- 2). Dev. Biol. 157:28-37.

27. Sugi, Y., and Lough, J. 1995. Activin-A and FGF-2 mimic the inductive effects of anterior endoderm on terminal cardiac myogenesis in vitro. Dev. Biol. 168:567-574.

28. Solloway, M.J., and Harvey, R.P. 2003. Molecular pathways in myocardial development: a stem cell perspective. Cardiovasc. Res. 58:264-277.

29. Liu, Y.J., et al. 2004. Hemangiopoietin, a novel human growth factor for the primitive cells of both hematopoietic and endothelial cell lineages. Blood. 103:4449-4456.

30. Burger, P.E., et al. 2002. Fibroblast growth factor receptor- 1 is expressed by endothelial progenitor cells. Blood. 100:3527-3535.

31. Kashiwakura, I., and Takahashi, T.A. 2003. Basic fibroblast growth factor-stimulated ex vivo expansion of haematopoietic progenitor cells from human placental and umbilical cord blood. Br. J. Haematol. 122:479-488.

32. Wiesel, P., Mazzolai, L., Nussberger, J., and Pedrazzini, T. 1997. Two-kidney, one clip and onekidney, one clip hypertension in mice. Hypertension. 29:1025-1030.

33. Nocka, K., et al. 1989. Expression of c-kit gene products in known cellular targets of W mutations in normal and W mutant mice: evidence for an impaired c-kit kinase in mutant mice. Genes Dev. 3:816-826.

34. Nocka, K., et al. 1990. Molecular bases of dominant negative and loss of function mutations at the murine c-kit/white spotting locus: W37, Wv, W41 and W. EMBO J. 9:1805-1813.

35. McKinney-Freeman, S.L., et al. 2002. Musclederived hematopoietic stem cells are hematopoietic in origin. Proc. Natl. Acad. Sci. U. S. A 99:1341-1346

36. Mery, A., et al. 2003. Commitment of embryonic stem cells toward a cardiac lineage: molecular mechanisms and evidence for a promising thera- peutic approach for heart failure. J. Muscle Res. Cell Motil. 24:269-274.

37. Roell,W., et al. 2002. Cellular cardiomyoplasty improves survival after myocardial injury. Circulation. 105:2435-2441.

38. Behfar, A., et al. 2002. Stem cell differentiation requires a paracrine pathway in the heart. FASEB J. 16:1558-1566.

39. Fijnvandraat, A.C., et al. 2003. Cardiomyocytes purified from differentiated embryonic stem cells exhibit characteristics of early chamber myocardium. J. Mol. Cell. Cardiol. 35:1461-1472.

40. Pera, M.F., et al. 2003. Isolation, characterization, and differentiation of human embryonic stem cells. Methods Enzymol. 365:429-446.

41. Alison, M.R., Poulsom, R., Forbes, S., and Wright, N.A. 2002. An introduction to stem cells. J. Pathol. 197:419-423.

42. Morabito, C.J., Dettman, R.W., Kattan, J., Collier, J.M., and Bristow, J. 2001. Positive and negative regulation of epicardial-mesenchymal transformation during avian heart development. Dev. Biol. 234:204-215.

43. Wessels, A., and Perez-Pomares, J.M. 2004. The epicardium and epicardially derived cells (EPDCs) as cardiac stem cells [review]. Anat. Rec. A Discov. Mol. Cell. Evol. Biol. 276A:43-57.

44. Thiery, J.P. 2003. Epithelial-mesenchymal transitions in development and pathologies. Curr. Opin. Cell Biol. 15:740-746.

45. Sefton, M., Sanchez, S., and Nieto, M.A. 1998 Conserved and divergent roles for members of the Snail family of transcription factors in the chick and mouse embryo. Development. 125:3111-3121.

46. Ciruna, B., and Rossant, J. 2001. FGF signaling regulates mesoderm cell fate specification and morphogenetic movement at the primitive streak. Dev. Cell. 1:37-49.

47. Potts, J.D., and Runyan, R.B. 1989. Epithelialmesenchymal cell transformation in the embryonic heart can be mediated, in part, by transforming growth factor beta. Dev. Biol. 134:392-401.

48. Yuan, S., and Schoenwolf, G.C. 2000. Islet-1 marks the early heart rudiments and is asymmetrically expressed during early rotation of the foregut in the chick embryo. Anat. Rec. 260:204-207.

49. Cai, C.L., et al. 2003. Isl1 identifies a cardiac progenitor population that proliferates prior to differentiation and contributes a majority of cells to the heart. Dev. Cell. 5:877-889

50. Laugwitz, K.L., et al. 2005. Postnatal isl1+ cardioblasts enter fully differentiated cardiomyocyte lineages. Nature. 433:647-653.

51. Stanford, W.L., et al. 1997. Altered proliferative response by $\mathrm{T}$ lymphocytes of Ly-6A (Sca-1) null mice. J. Exp. Med. 186:705-717.

52. Ito, C.Y., Li, C.Y., Bernstein, A., Dick, J.E., and Stanford, W.L. 2003. Hematopoietic stem cell and progenitor defects in Sca-1/Ly-6A-null mice. Blood. 101:517-523

53. Bonyadi, M., et al. 2003. Mesenchymal progenitor self-renewal deficiency leads to age-dependent osteoporosis in Sca-1/Ly-6A null mice. Proc. Natl. Acad. Sci. U. S. A. 100:5840-5845.

54. Oh, H., et al. 2003. Cardiac progenitor cells from adult myocardium: homing, differentiation, and fusion after infarction. Proc. Natl. Acad. Sci. U. S. A. 100:12313-12318.

55. Beltrami, A.P., et al. 2003. Adult cardiac stem cells are multipotent and support myocardial regeneration. Cell. 114:763-776.

56. Messina, E., et al. 2004. Isolation and expansion of adult cardiac stem cells from human and murine heart. Circ. Res. 95:911-921.

57. Hara, M., et al. 2002. Evidence for a role of mast cells in the evolution to congestive heart failure. J. Exp. Med. 195:375-381.

58. Jiang, Y., et al. 2002. Pluripotency of mesenchymal stem cells derived from adult marrow. Nature. 418:41-49.

59. Barron, M., Gao, M., and Lough, J. 2000. Requirement for BMP and FGF signaling during cardiogenic induction in non-precardiac mesoderm is specific, transient, and cooperative. Dev. Dyn. 218:383-393.

60. Sugi, Y., and Markwald, R.R. 2003. Endodermal growth factors promote endocardial precursor cell formation from precardiac mesoderm. Dev. Biol. 263:35-49.

61. Schultz, J.E., et al. 1999. Fibroblast growth factor-2 mediates pressure-induced hypertrophic response. J. Clin. Invest. 104:709-719.

62. Torella, D., et al. 2004. Cardiac stem cell and myocyte aging, heart failure, and insulin-like growth factor-1 overexpression. Circ. Res. 94:514-524.

63. Okabe, M., Ikawa, M., Kominami, K., Nakanishi, T., and Nishimune, Y. 1997. 'Green mice' as a source of ubiquitous green cells. FEBS Lett. 407:313-319. 